\title{
Prior-based Piecewise-smooth Segmentation by Template Competitive Deformation using Partitions of Unity
}

\author{
Oudom Somphone ${ }^{1,2}$, Benoit Mory ${ }^{1}$, \\ Sherif Makram-Ebeid ${ }^{1}$, and Laurent Cohen $^{2}$ \\ 1 Medisys Research Lab, Philips Healthcare \\ 33 rue de Verdun, B.P. 313, F-92156 Suresnes Cedex, France \\ \{oudom.somphone, benoit.mory, sherif.makram-ebeid\}@philips.com \\ 2 CEREMADE, CNRS UMR 7534, Université Paris Dauphine, France \\ cohen@ceremade.dauphine.fr
}

\begin{abstract}
We propose a new algorithm for two-phase, piecewise-smooth segmentation with shape prior. The image is segmented by a binary template that is deformed by a regular geometric transformation. The choice of the template together with the constraint on the transformation introduce the shape prior. In particular, the topology of the shape is preserved if the transformation is diffeomorphic. The deformation is guided by the maximization of the likelihood of foreground and background intensity models, so that we can refer to this approach as Competitive Deformation. In each region, the intensity is modelled as a smooth approximation of the original image. We represent the transformation using a Partition of Unity Finite Element Method, which consists in representing each component with polynomial approximations within local patches. A conformity constraint between the patches provides a way to control the globality of the deformation. We show several results on synthetic images, as well as on medical data from different modalities.
\end{abstract}

\section{Introduction}

Image segmentation is a fundamental topic in computer vision, which has motivated many works to cope with challenging issues such as noise, occlusions and low contrasted regions. A common approach is the introduction of prior knowledge in order to constrain the solution to remain close to a given class of shapes. Statistical models have been proposed, using for example Principal Component Analysis (PCA), like the well-known active shape models [1]. Such techniques require careful training in order to capture the variability of the shapes, and do not enable segmentation if no training database is available.

Shape priors have also been incorporated in the level set framework via an additive shape term in the energy, that penalizes the dissimilarity between the level set function for segmentation and the one embedding the prior shape [2-5]. In the model of Leventon et al. [6] and further works along the same line [7-9], 
a PCA of training shapes embedded in level set functions is computed in order to define a linear statistical model for the shape term. Non-linear versions have also been explored [10]. For some applications, those models may however suffer from the uncontrollable topology changes allowed by the level set representation, which is most often not desirable when one wants to impose a shape prior.

A possible alternative to control the topology is to directly apply a geometric deformation to the prior shape, provided that the transformation is diffeomorphic $[11,12]$. Two-phase segmentation is performed by deforming a binary template towards the image. The prior is the template itself, and the shape constraint is conveyed through the choice of a class of allowed deformations. This choice is crucial: excessive constraint may result in poor segmentation results, whereas insufficient constraint may lead to a final shape too far from the prior.

The deformation can be guided by the maximization of the likelihood of userdefined intensity models, given the pixel values observed in both the foreground and the background. The approach bears similarities with Region Competition techniques [13], with the significant difference that the unknown variable is not the partitioning itself but the deformation of a predefined template. We will refer to this method as Competitive Deformation. Obviously, the choice of appropriate region intensity models is also essential. On one hand, piecewise-constant and global models are simple but their applicability is limited. On the other hand, piecewise-smooth and local models are more relevant in many cases, at the cost of an increased computational complexity.

We propose a variational formulation of two-phase, piecewise-smooth segmentation based on Competitive Deformation and a Partition of Unity Finite Element Method (PUFEM). The key idea is to represent the deformation field with polynomial approximations within overlapping local patches. The method includes a conformity constraint between the patches, which offers a good control over the range of the smoothness, and hence over the strength of the prior. Moreover, this framework provides efficient smooth representations of the region intensity models that naturally extrapolate beyond the boundary.

This paper is organised as follows. In section 2, we first give the basic formulation for two-phase segmentation by template competitive deformation. Then in section 3, we give a brief overview of the PUFEM framework before presenting our formulation of piecewise-smooth segmentation in section 4 . In section 5 , we show experimental results on illustrative synthetic images, and on medical images.

\section{Basic formulation of Competitive Deformation}

Let the open bounded set $\Omega \subset \mathbb{R}^{d}$ be the domain of a real-valued image $I$. Twophase segmentation aims at partitioning $\Omega$ into a foreground and a background that are homogeneous in terms of intensity properties. In the classical level set version of Region Competition [13], the optimal partition of $\Omega$ is obtained by evolving a level set function that embeds the region boundary, allowing unde- 
sirable topology changes in the case of prior-based segmentation. To control the topology of the result, we rather deform the characteristic function $\chi$ of a prior foreground region $\Sigma$ :

$$
\chi(\mathbf{x})= \begin{cases}1 & \text { if } \mathbf{x} \in \Sigma \\ 0 & \text { otherwise }\end{cases}
$$

with a diffeomorphic geometric transformation $\psi: \Omega \rightarrow \psi(\Omega)$. The basic form of the two-phase competitive deformation problem reads:

$$
\min _{\psi, \boldsymbol{\alpha}_{1}, \boldsymbol{\alpha}_{2}}\left\{\int_{\mathbf{x} \in \Omega} \chi \circ \psi(\mathbf{x}) r_{1}\left(\boldsymbol{\alpha}_{1}, \mathbf{x}\right)+\int_{\mathbf{x} \in \Omega}(1-\chi \circ \psi(\mathbf{x})) r_{2}\left(\boldsymbol{\alpha}_{2}, \mathbf{x}\right)+\gamma \mathcal{R}(\psi)\right\}
$$

where the $r_{i}: \Omega \rightarrow \mathbb{R}$ are the region model functions that encode the intensity properties in the foreground and the background. Each $r_{i}$ usually depends on a set of parameters $\boldsymbol{\alpha}_{i}$, e.g. the mean intensity value, the standard deviation, etc. $\mathcal{R}$ is the regularization constraint on $\psi$ and $\gamma$ a positive constant that controls its weighting. The minimization is carried out iteratively by alternating the following two steps:

(A) Considering the transformation $\psi$ fixed, optimize and update the model's parameters $\boldsymbol{\alpha}_{1}$ and $\boldsymbol{\alpha}_{2}$,

(B) Considering $\boldsymbol{\alpha}_{1}$ and $\boldsymbol{\alpha}_{2}$ fixed, minimize w.r.t $\psi$.

\subsection{Region intensity models}

In this formulation, simple global statistics can be used to represent the region intensity properties, such as the well-known piecewise-constant case $[14,15]$, assuming Gaussian intensity distributions with known variance, i.e.:

$$
r_{i}\left(m_{i}, \mathbf{x}\right)=\left(I(\mathbf{x})-m_{i}\right)^{2}
$$

$m_{i}$ being the mean intensity value in region $i$. The Gaussian assumption has practical limitations and is not valid in images showing more complex intensity distributions. In a similar template deformation context, Saddi et al. [12] used the more general non-parametric model:

$$
r_{i}\left(p_{i}, \mathbf{x}\right)=-\log p_{i}(I(\mathbf{x}))
$$

where $p_{i}$ is the intensity probability density function in region $i$. Estimating global probability densities for the foreground and background using the whole image still has limitations in practice. Especially critical are the cases of cluttered and heterogeneous backgrounds and the presence of low-frequency artifacts such as illumination changes. Moreover, it may be difficult to obtain a precise positioning of the boundary since the local contributions of nearby pixels from both sides are diluted in the global estimation of the densities. 
To overcome these limitations, it is natural to turn to local models, assuming smoothly-varying intensity distributions. In the Gaussian case with spacedependent mean values, this leads to piecewise-smooth segmentation. The error function reads:

$$
r_{i}\left(I_{i}, \mathbf{x}\right)=\left(I(\mathbf{x})-I_{i}(\mathbf{x})\right)^{2}+\mu_{i}\left|\nabla I_{i}(\mathbf{x})\right|^{2}
$$

where $I_{i}$ becomes a function that approximates $I$ and is constrained to be smooth inside region $i ; \mu_{i}$ is the smoothing parameter. Vese and Chan [16], and simultaneously Tsai et al. [17], introduced a level-set formulation based on diffusion and on the work of Mumford and Shah [18]. This diffusion scheme requires the iterative resolution of a PDE with new boundary conditions at each update step (B), which is computationnally costly. Methods based on Gaussian convolution were recently proposed [19-21], giving qualitatively similar results in a more efficient way. They enable the extrapolation of the model beyond the region boundary, to an extent that depends on the kernel's scale. In section 4 , we present an alternative method based on finite elements with polynomial extrapolary properties.

\subsection{Deformation models}

Compliance with the shape prior is determined by the class of deformations allowed by the regularization constraint $\mathcal{R}$. Therefore it has to be chosen carefully. In particular, $\mathcal{R}$ must take into account the fact that a shape is invariant to some geometric, global transformations such as translation, rotation, scaling, shearing, etc. For example, several instances of a same shape are shown on Fig. 1. Despite
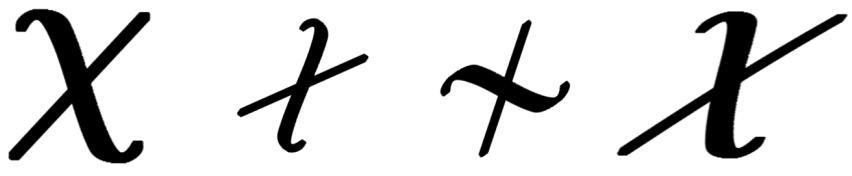

Fig. 1. Several instances of a same shape

their different positions, orientations and sizes, they all represent the same shape. Consequently, a relevant regularizer $\mathcal{R}$ shall not penalize such transformations.

Within the template matching context, a similarity transformation is used in [15] to segment synthetic images, excluding more complex deformations; in the non-rigid case, Saddi et al. [12] constrain the deformation with a diffeomorphic fluid model, thus enabling the result to strongly deviate from the prior shape.

Methods based on local basis expansions of the deformation such as B-splines $[22,23]$ or Radial Basis Functions $[24,25]$ provide a suitable compromise between global and fluid models. As will be seen in the subsequent sections, we use a finite element registration framework based on a partition of unity, which enables to easily control the globality of $\psi$. Moreover, unlike Free Form Deformation methods, our regularization term does not penalize globally polynomial transformations. 


\section{Partition of Unity Finite Element Representation}

In this section, we give an overview of the mathematical framework of the PUFEM [26] that we use to represent a given scalar function: in our case, the approximation images $I_{i}$ in each region, and the components of the transformation $\psi$ in each dimension. The basic idea is to locally fit the said scalar field with $d$-dimensional polynomials and smoothly blend them afterwards to obtain a regular representation.

Let $F$ be a real-valued function defined on $\Omega$. We define a set $\mathcal{N}$ of nodes distributed over $\Omega$. A node $n \in \mathcal{N}$ is characterized by:

- a point $\mathbf{c}^{(n)} \in \Omega$, called center of the node,

- an open bounded subdomain $\Omega^{(n)} \subset \mathbb{R}^{d}$ containing $\mathbf{c}^{(n)}$, called patch,

- a function $\varphi^{(n)}: \mathbb{R}^{d} \rightarrow \mathbb{R}$, called PU-function,

- a set of $\rho^{(n)}$ functions $\mathcal{B}^{(n)}=\left\{p_{r}^{(n)}: \Omega \rightarrow \mathbb{R} \mid r \leq \rho^{(n)}\right\}$, called the local basis.

We allow the patches to overlap and assume the families $\left(\Omega^{(n)}\right)_{n \in \mathcal{N}}$ and $\left(\varphi^{(n)}\right)_{n \in \mathcal{N}}$ to fullfil the Partition of Unity conditions:

$$
\Omega \subset \bigcup_{n \in \mathcal{N}} \Omega^{(n)} \quad \text { and } \quad \forall \mathbf{x} \in \Omega \quad \sum_{n \in \mathcal{N}} \varphi^{(n)}(\mathbf{x})=1
$$

For the sake of computational efficiency, our nodes are distributed over a regular, rectangular array and each patch $\Omega^{(n)}$ is a cuboid centered on $\mathbf{c}^{(n)}$. This configuration is illustrated on Fig. 2.a. The PU-function $\varphi^{(n)}$ has a compact support included in $\Omega^{(n)}$; it is non-negative, equal to 1 at $\mathbf{c}^{(n)}$ and vanishes with the

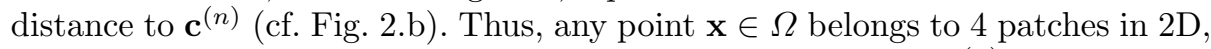
and 8 patches in $3 \mathrm{D}$, with weights given by the corresponding $\varphi^{(n)}(\mathbf{x})$. The basis

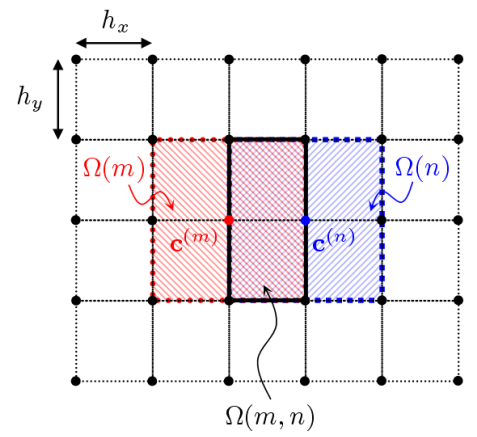

(a) Nodes and patches

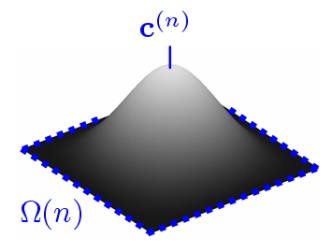

(b) PU-function

Fig. 2. Example of Partition of Unity configuration in 2D.

functions $p_{r}^{(n)}$ are the monomials of all degrees up to a user-defined maximum 
degree - e.g. in 2D and degrees up to $2: 1, x, y, x^{2}, x y, y^{2}$ - centered on $\mathbf{c}^{(n)}$, so that $F$ is locally modelled at node $n$ by a polynomial $\widetilde{F}^{(n)}$ :

$$
\widetilde{F}^{(n)} \widehat{=} \sum_{r \leqslant \rho^{(n)}} a_{r}^{(n)} p_{r}^{(n)}
$$

where the $a_{r}^{(n)}$ are real coefficients. The global representation is then constructed by blending the $\widetilde{F}^{(n)}$ with the PU-functions:

$$
\widetilde{F}=\sum_{n \in \mathcal{N}} \varphi^{(n)} \widetilde{F}^{(n)}=\sum_{n \in \mathcal{N}} \sum_{r \leqslant \rho^{(n)}} \varphi^{(n)} a_{r}^{(n)} p_{r}^{(n)}
$$

According to (8), $\widetilde{F}^{(n)}$ is as regular as the PU-functions per se. However, we want to impose a controllable, "long range" regularization, or rather, globality. To this end, we introduce the notion of non-conformity between two neighbouring nodes $m$ and $n$ through the energy:

$$
\mathcal{S}_{\kappa}^{(m, n)}(\widetilde{F})=\int_{\Omega^{(m, n)}} \sum_{|\boldsymbol{\beta}| \leqslant \kappa} \varphi^{(m)} \varphi^{(n)}\left(D^{\boldsymbol{\beta}} \widetilde{F}^{(m)}-D^{\boldsymbol{\beta}} \widetilde{F}^{(n)}\right)^{2}
$$

where $\boldsymbol{\beta}=\left(\beta_{1}, \beta_{2}, \ldots, \beta_{k}\right)$ and $D^{\boldsymbol{\beta}}$ is the partial derivative operator in the standard multi-index notations. This local energy has an intuitive interpretation: it penalizes $\widetilde{F}$ if its local representations at nodes $m$ and $n$ and their derivatives up to order $\kappa$ differ in the overlapping region $\Omega^{(m, n)}$. The total conformity energy is then defined by:

$$
\mathcal{S}_{\kappa}(\widetilde{F})=\frac{1}{2} \sum_{n \in \mathcal{N}} \sum_{m \in \mathcal{V}(n)} \mathcal{S}_{\kappa}^{(m, n)}(\widetilde{F})
$$

where $\mathcal{V}^{(n)}$ is the set of neighbours of node $n$ in 4-connexity. This inter-node conformity constraint is a key feature of our method. It enables smooth representations of the region intensity models, that are naturally extrapolated beyond the boundary. As for the representation of the deformation, this energy is zero when all the local representations are equal, i.e. when $\psi$ is globally polynomial. Thus, in the case of local affine bases, global translation, rotation, scaling and shearing are not penalized.

\section{Piecewise-smooth segmentation with Competitive Deformation}

We now detail our two-phase, piecewise-smooth segmentation formulation casting the Competitive Deformation problem into the PUFEM framework.

\subsection{Our formulation}

We address the deformation of the template characteristic function $\chi$ through its corresponding displacement vector field $\mathbf{u}=\psi-\mathbf{i d}$, the components of which 
are represented as in (8) by sets of coefficients that we pile up into one vector $\mathbf{a}$. The regularization term $\mathcal{R}(\psi)$ in $(2)$ takes the form of an inter-node conformity constraint $S_{\kappa}(\mathbf{a})$, defined as the sum of the constraints on each component of $\mathbf{u}$.

In region $i, I$ is approximated by a locally polynomial image $I_{i}^{\boldsymbol{\alpha}_{i}}$, represented over a set of nodes $\mathcal{N}_{i}$ by a set of coefficients $\boldsymbol{\alpha}_{i}$ according to (8) and (7):

$$
I_{i}^{\boldsymbol{\alpha}_{i}}=\sum_{n \in \mathcal{N}_{i}} \varphi_{i}^{(n)} I_{i}^{(n)} \quad \text { where } \quad I_{i}^{(n)}=\sum_{r \leqslant \rho_{i}^{(n)}} \alpha_{i r}^{(n)} p_{i r}^{(n)}
$$

Most existing piecewise-smooth methods include regularization inside each phase (e.g. see (5)). Consequently, $I_{i}$ is not explicitly defined outside region $i$. We apply an inter-node conformity constraint $\mathcal{S}_{\kappa_{i}}\left(\boldsymbol{\alpha}_{i}\right)$ involving all the nodes of $\mathcal{N}_{i}$, so that our energy functional reads:

$$
\begin{aligned}
\mathcal{E}\left(\mathbf{a}, \boldsymbol{\alpha}_{1}, \boldsymbol{\alpha}_{2}\right) & =\int_{\Omega} \chi \circ \psi^{\mathbf{a}}\left(I-I_{1}^{\boldsymbol{\alpha}_{1}}\right)^{2}+\int_{\Omega}\left(1-\chi \circ \psi^{\mathbf{a}}\right)\left(I-I_{2}^{\boldsymbol{\alpha}_{2}}\right)^{2} \\
& +\gamma \mathcal{S}_{\kappa}(\mathbf{a})+\mu_{1} \mathcal{S}_{\kappa_{1}}\left(\boldsymbol{\alpha}_{1}\right)+\mu_{2} \mathcal{S}_{\kappa_{2}}\left(\boldsymbol{\alpha}_{2}\right)
\end{aligned}
$$

We minimize (12) by alternating steps (A) and (B) (see section 2) until convergence.

\subsection{Step (A): Estimating the image approximations}

Minimization w.r.t. the parameter set $\boldsymbol{\alpha}_{i}$ is achieved, considering the energy:

$$
\mathcal{E}_{i}\left(\boldsymbol{\alpha}_{i}\right)=\int_{\Omega} \chi_{i}\left(I-I_{i}^{\boldsymbol{\alpha}_{i}}\right)^{2}+\mu_{i} \mathcal{S}_{\kappa_{i}}\left(\boldsymbol{\alpha}_{i}\right)
$$

where we define $\chi_{1}=\chi \circ \psi$ and $\chi_{2}=1-\chi \circ \psi$. The first term is a masked least square term that enforces $I_{i}$ to fit the image within region $i$. The second term is an inter-node conformity constraint that compels $I_{i}$ to be regular everywhere in $\Omega$. In other words, $I_{i}$ results from a regularized approximation of $I$ inside region $i$, and since no fitting constraint is imposed outside, it is extrapolated beyond the region border by regularization only.

$\mathcal{S}_{\kappa_{i}}$ is a quadratic function of the parameters $\boldsymbol{\alpha}_{i}$ and hence so is $\mathcal{E}_{i}$. Minimization is then achieved by classical linear regression. Saying that the derivatives of $\mathcal{E}_{i}$ w.r.t. the $\alpha_{i r}^{(n)}$ vanish provides a system of linear equations which is sparse, since the nodes are only related to each other in 4-connexity, due to the overlap pattern between the patches (see Fig. 2.a). More precisely, we need to solve:

$$
\mathbf{M}^{i} \cdot \boldsymbol{\alpha}_{i}=\mathrm{g}^{i}
$$

where $\mathbf{M}^{i}$ is a symmetric, non-negative definite matrix of size $\sum_{n} \rho_{i}^{(n)}$, and $\mathbf{g}^{i}$ a vector of length $\sum_{n} \rho_{i}^{(n)}$. Their entries are given in the appendix. We use a Conjugate Gradient descent, well-suited for solving sparse linear systems [27]. 


\subsection{Step (B): Template Registration}

We show that step (B) is equivalent to a classical registration problem based on a Sum of Square Difference criterion. Let $\hat{\chi}$ be the signed characteristic function of the prior foreground $\Sigma$ :

$$
\hat{\chi}(\mathbf{x})=\left\{\begin{aligned}
1 & \text { if } \mathbf{x} \in \Sigma \\
-1 & \text { otherwise }
\end{aligned}\right.
$$

Then we can replace $\chi$ by $(1+\hat{\chi}) / 2$ in (12). The region parameters $\boldsymbol{\alpha}_{i}$ being fixed, the energy to minimize w.r.t. $\mathbf{a}$ is:

$$
-\frac{1}{2} \int_{\Omega}\left(\hat{\chi} \circ \psi^{\mathbf{a}}\right) r+\gamma \mathcal{S}_{\kappa}(\mathbf{a})
$$

where $r=\left(I-I_{2}^{\boldsymbol{\alpha}_{2}}\right)^{2}-\left(I-I_{1}^{\boldsymbol{\alpha}_{1}}\right)^{2}$. By writing:

$$
\hat{\chi} \circ \psi^{\mathbf{a}} r=-\frac{1}{2}\left(\left(\hat{\chi} \circ \psi^{\mathbf{a}}-r\right)^{2}-\left(\hat{\chi} \circ \psi^{\mathbf{a}}\right)^{2}-r^{2}\right)
$$

and since $\left(\hat{\chi} \circ \psi^{\mathbf{a}}\right)^{2}=1$ and $r^{2}$ is independent of the parameters $\mathbf{a}$, we can reduce the energy to minimize to:

$$
\mathcal{E}_{u}(\mathbf{a})=\frac{1}{4} \int_{\Omega}\left(\hat{\chi} \circ \psi^{\mathbf{a}}-r\right)^{2}+\gamma \mathcal{S}_{\kappa}(\mathbf{a})
$$

Therefore step (B) boils down to an SSD-based registration problem, with $r$ being the reference and $\hat{\chi}$ the template. Minimization of $\mathcal{E}_{u}$ follows a global-tolocal strategy: we define a coarse-to-fine dyadic pyramid of node distributions. Once the solution has been computed at one level, it is projected on the basis of the next finer level to provide an initialization. A detailed description of the minimization scheme is out of the scope of this paper and can be found in [28].

\section{Results and discussion}

We first present results on illustrative synthetic, noisy images (see Fig. 3). The first image, " $\chi$ ", consists of two heterogeneous phases. The results show the robustness of our method to noise and strong intensity variations. The extrapolatory property is illustrated by figure (d) of the first line. The second image, "Treble Clef", consists of a homogeneous foreground on a heterogeneous background and contains occluding objects. The final segmentations are robust to occlusions and leaks.

We then apply our method to segment heart chambers on cardiac ultrasound (Fig. 4) and cine MR (Fig. 5) images. The final deformations are well-constrained so that the topologies of the prior shapes are preserved. On the MR images, the foreground region shall include the papillary muscles when segmenting the blood pool, which is challenging as they appear darker an may be confused with the myocardium. 


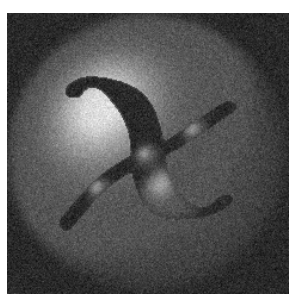

" $\chi "$ and its prior shape
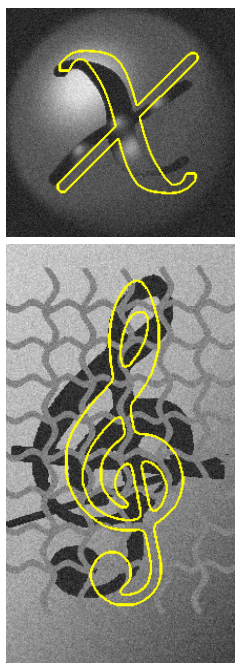

(a)
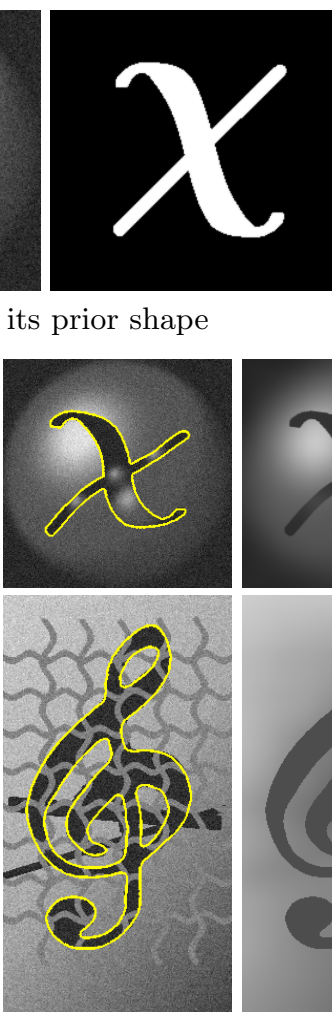

(b)

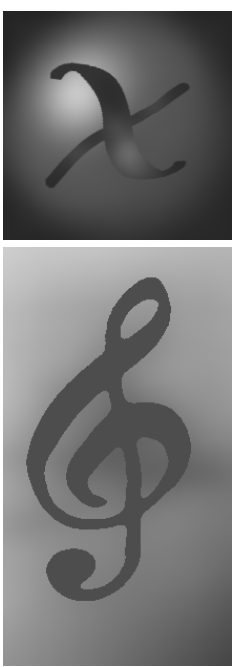

(c)
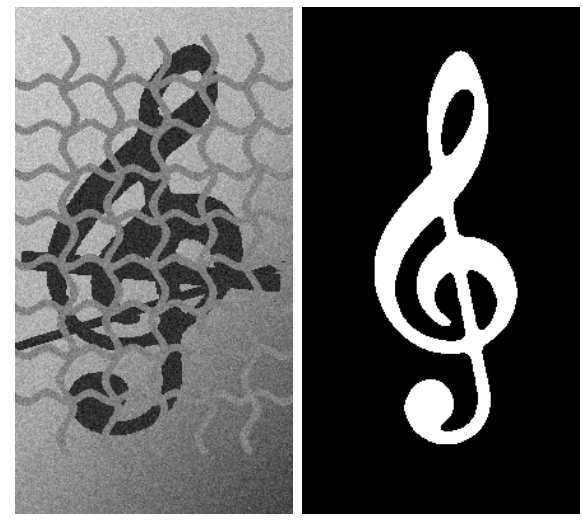

"Treble Clef" and its prior shape

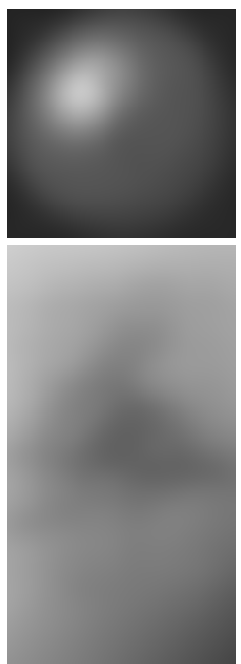

(d)
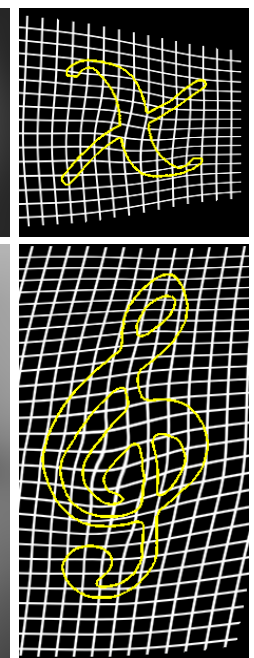

(e)

Fig. 3. Synthetic images " $\chi$ " and "Treble Clef": (a) Initializations. (b) Final segmentation. (c) Final piecewise-smooth approximations $\chi I_{1}+(1-\chi) I_{2}$. (d) Final background approximations $I_{2}$. (e) Final deformations. 

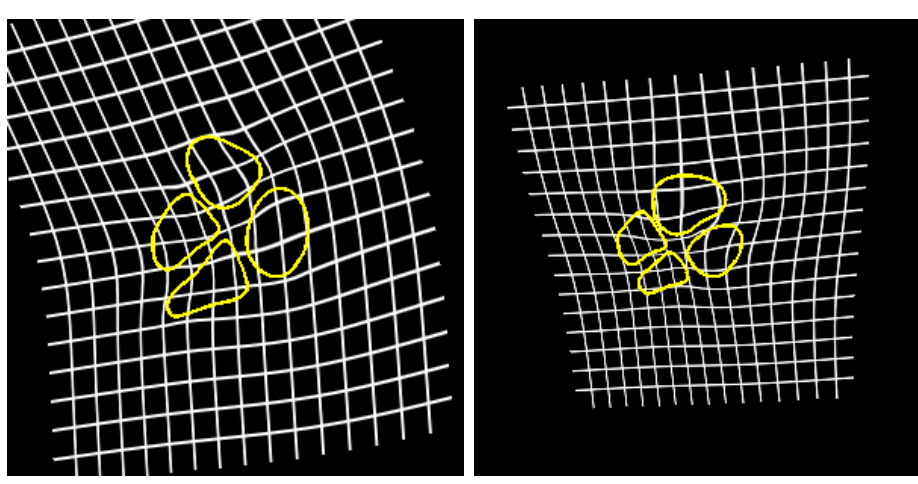

o
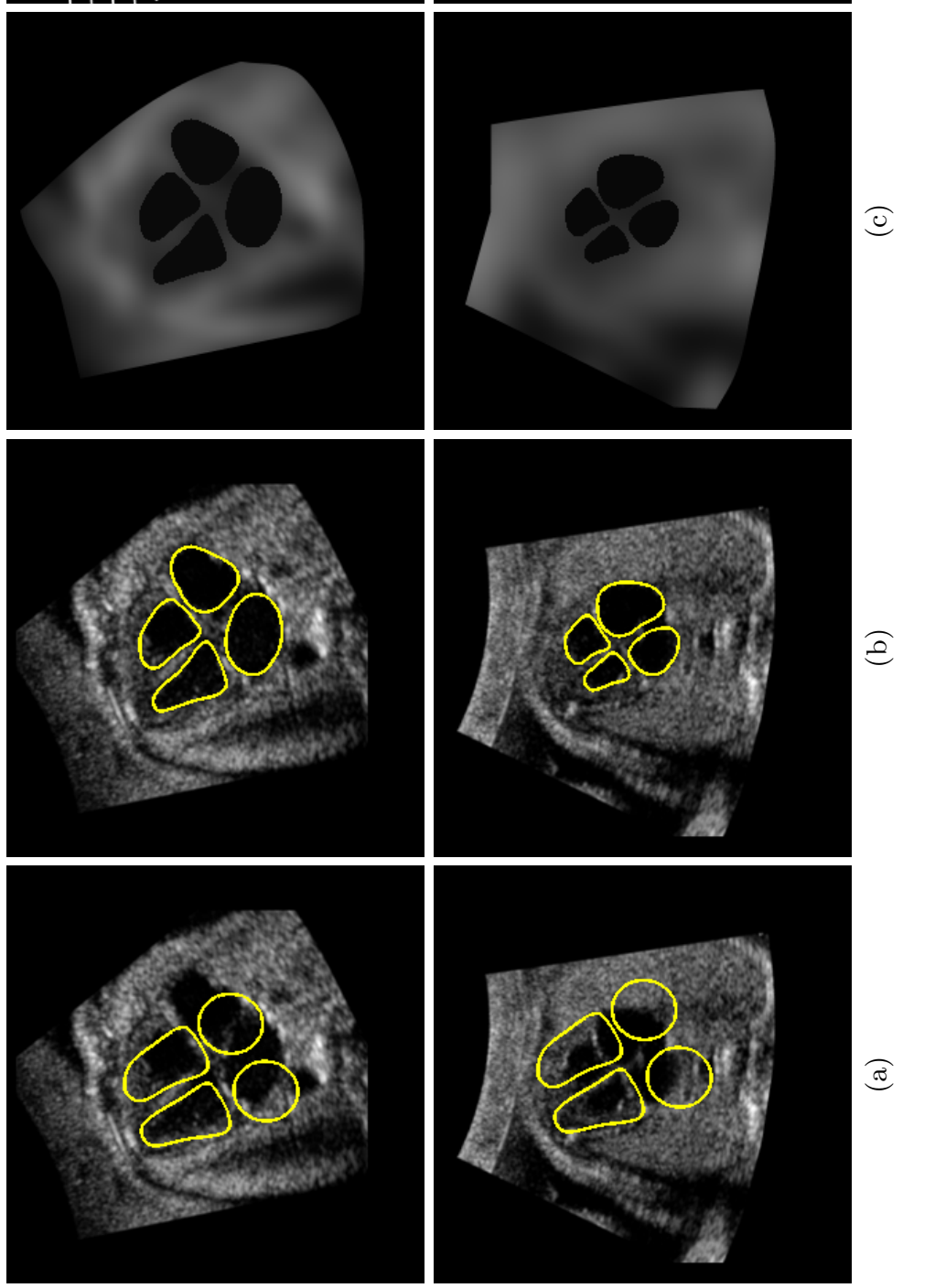

e

造

ฮ. สี

昰

$>\dot{1}$

宊

ป

章 +

핮

की

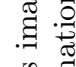

. ב

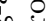

을 을

쿵

迅

( $)$

.].ّ.

2.

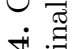

$\underset{0}{+\infty}$ 

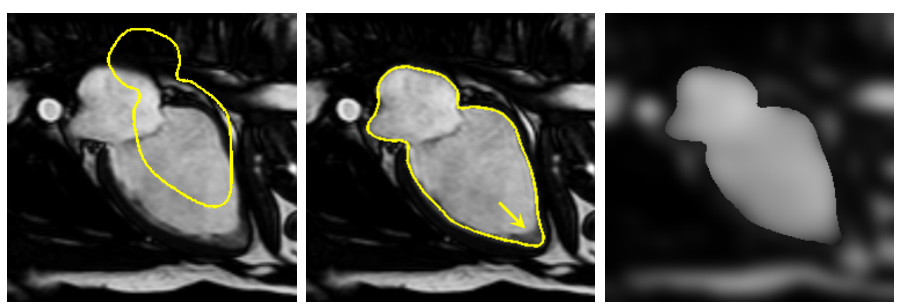

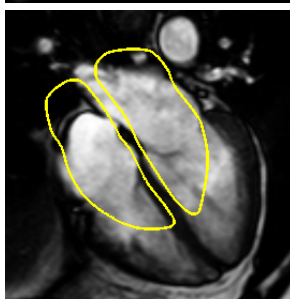

(a)

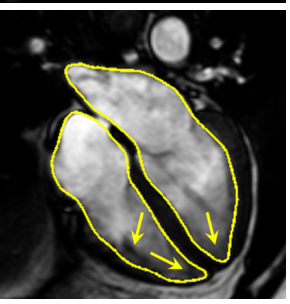

(b)

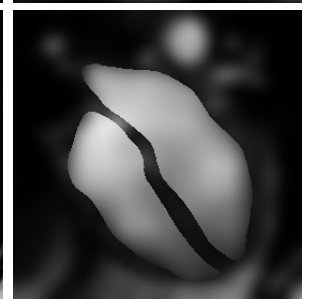

(c)

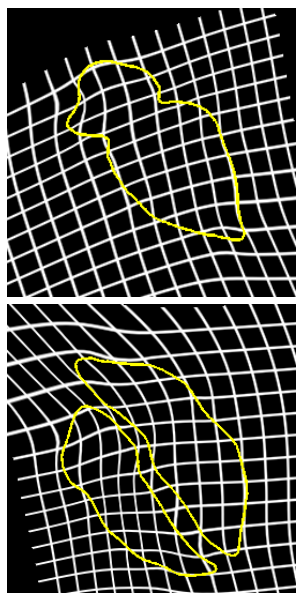

(d)

Fig. 5. Cardiac MR long-axis images, two-chamber (first line) and four-chamber (second line) views: (a) Initializations. (b) Final segmentations. (c) Final piecewise-smooth approximations $\chi I_{1}+(1-\chi) I_{2}$. (d) Final deformations. Papillary muscles are pointed at by arrows.

On Fig. 6 we compare three deformation models: affine, PUFEM, and fluid. An affine deformation (a) is obviously too restricted to obtain an accurate segmentation from the prior shape that is used (Fig. 5.a bottom). On the opposite, the fluid model (c) allows strong local deformations and hence strong deviations from the prior shape. This is visible on the lower part of the right ventricle segmentation where we can see a curvature inversion when compared to the prior, and a consequent exclusion of the papillary muscles. The PUFEM model (b) provides a good compromise to have both accuracy and compliance with the prior shape.

\section{Conclusion}

We introduced a novel variational approach for two-phase, piecewise-smooth image segmentation based on prior shape Competitive Deformation. We cast our formulation into the Partition of Unity Finite Element framework, motivated by its long-range regularization properties, suitable for the class of transformations that is needed to abide by the shape prior. Indeed, the inter-node conformity constraint provides a good control over the globality of the deformation and does not penalize basic global transformations. This framework is also well-adapted for representing the region intensity models as smooth approximations of the original image on its whole domain. Our algorithm was successfully applied to challenging synthetic images and to medical images, with robustness to noise, occlusions and leaks. 


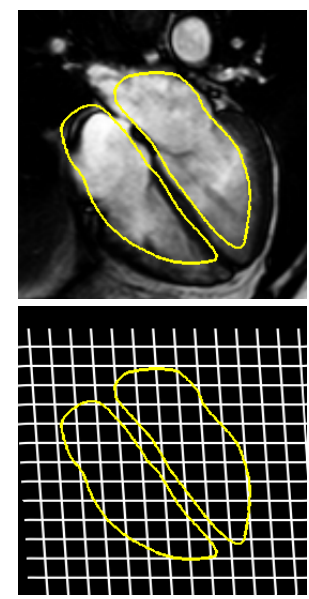

(a)

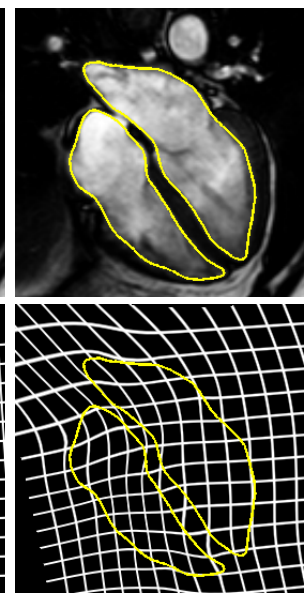

(b)

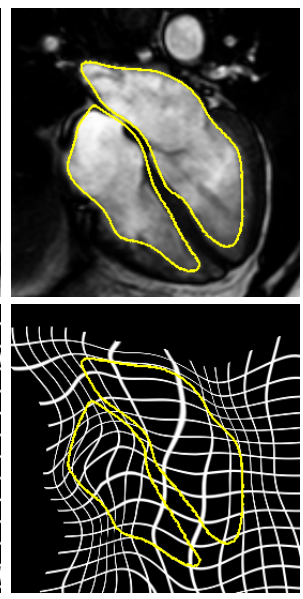

(c)

Fig. 6. Comparison between three deformation models. Final segmentations are displayed on the first line and final deformations on the second line. (a) Affine model. (b) PUFEM model. (c) Affine + fluid model [12].

\section{Appendix: entries of $\mathrm{M}^{i}$ and $\mathrm{g}^{i}$}

Estimating the image approximation in region $i$ (step (A) in the minimization of the functional (12)) boils down to the matricial equation:

$$
\mathbf{M}^{i} \cdot \boldsymbol{\alpha}_{i}=\mathbf{g}^{i}
$$

where $\mathbf{M}^{i}$ is a symmetric, non-negative definite matrix of size $\sum_{n} \rho_{i}^{(n)}$, and $\mathbf{g}^{i}$ a vector of length $\sum_{n} \rho_{i}^{(n)}$. Their entries are given by:

$$
\begin{aligned}
m_{(r, n)(s, m)}^{i} & =\int_{\Omega} \varphi_{i}^{(m)} \varphi_{i}^{(n)}\left(\chi_{i} p_{i s}^{(m)} p_{i r}^{(n)}-\mu_{i} \sum_{\boldsymbol{\beta} \leqslant \kappa_{i}} D^{\boldsymbol{\beta}} p_{i s}^{(m)} D^{\boldsymbol{\beta}} p_{i r}^{(n)}\right) \\
& +\delta_{m n} \mu_{i} \int_{\Omega} \varphi^{(n)} \sum_{\boldsymbol{\beta} \leqslant \kappa_{i}} D^{\boldsymbol{\beta}} p_{i s}^{(n)} D^{\boldsymbol{\beta}} p_{i r}^{(n)} \\
g_{(r, n)}^{i} & =\int_{\Omega} \chi_{i} \varphi_{i}^{(n)} p_{i r}^{(n)} I
\end{aligned}
$$

$\delta_{m n}$ being the Kronecker delta equal to 1 if $m=n$ and 0 otherwise. Since the support of a PU-function is included in the corresponding patch, $m_{(r, n)(s, m)}^{i}$ equals zero when $\Omega^{(m)} \cap \Omega^{(n)}=\varnothing$, i.e. when $m$ and $n$ are not neighbours in 4-connexity. Hence the sparseness of $\mathbf{M}^{i}$. 


\section{References}

1. Cootes, T.F., Taylor, C.J., Cooper, D.H., Graham, J.: Active shape models: Their training and application. Computer Vision and Image Understanding 61 (1995) $38-59$

2. Paragios, N., Rousson, M., Ramesh, V.: Matching distance functions: A shape-toarea variational approach for global-to-local registration. In: European Conference on Computer Vision. (2002) II: $775 \mathrm{ff}$.

3. Cremers, D., Sochen, N.A., Schnorr, C.: Towards recognition-based variational segmentation using shape priors and dynamic labeling. In: Scale Space. (2003) 388-400

4. Chan, T.F., Zhu, W.: Level set based shape prior segmentation. In: IEEE Computer Vision and Pattern Recognition or CVPR. (2005) II: 1164-1170

5. Raviv, T.R., Kiryati, N., Sochen, N.A.: Prior-based segmentation by projective registration and level sets. In: International Conference on Computer Vision. (2005) 204-211

6. Leventon, M.E., Grimson, W.E.L., Faugeras, O.D.: Statistical shape influence in geodesic active contours. In: IEEE Computer Vision and Pattern Recognition or CVPR. (2000) 316-323

7. Tsai, A., Yezzi, Jr., A.J., Wells, III, W.M., Tempany, C., Tucker, D., Fan, A., Grimson, W.E.L., Willsky, A.S.: Model-based curve evolution technique for image segmentation. In: IEEE Computer Vision and Pattern Recognition or CVPR. (2001) I:463-468

8. Tsai, A., Yezzi, A.J., Wells, III, W.M., Tempany, C., Tucker, D., Fan, A., Grimson, W.E.L., Willsky, A.S.: A shape-based approach to the segmentation of medical imagery using level sets. IEEE Trans. Medical Imaging 22 (2003) 137-154

9. Bresson, X., Vandergheynst, P., Thiran, J.P.: A variational model for object segmentation using boundary information and shape prior driven by the mumfordshah functional. International Journal of Computer Vision 68 (2006) 145-162

10. Cremers, D., Kohlberger, T., Schnorr, C.: Shape statistics in kernel space for variational image segmentation. Pattern Recognition 36 (2003) 1929-1943

11. Hong, B.W., Prados, E., Soatto, S., Vese, L.A.: Shape representation based on integral kernels: Application to image matching and segmentation. In: IEEE Computer Vision and Pattern Recognition or CVPR. (2006) I:833-840

12. Saddi, K.A., Chefd'hotel, C., Rousson, M., Cheriet, F.: Region-based segmentation via non-rigid template matching. In: Workshop on Mathematical Methods in Biomedical Image Analysis. (2007)

13. Zhu, S.C., Yuille, A.: Region competition: Unifying snakes, region growing, and bayes/mdl for multiband image segmentation. IEEE Transactions on Pattern Analysis and Machine Intelligence 18 (1996) 884-900

14. Chan, T., Vese, L.: Active contours without edges. IEEE Trans. on Image Processing 10 (2001) 266-277

15. An, J.H., Chen, Y.: Region based image segmentation using a modified mumfordshah algorithm. In: Scale Space and Variational Methods in Computer Vision. (2007) 733-742

16. Vese, L.A., Chan, T.F.: A multiphase level set framework for image segmentation using the mumford and shah model. International Journal of Computer Vision $\mathbf{5 0}$ (2002) 271-293

17. Tsai, A., Yezzi, Jr., A.J., Willsky, A.S.: Curve evolution implementation of the Mumford-Shah functional for image segmentation, denoising, interpolation, and magnification. IEEE Trans. Image Processing 10 (2001) 1169-1186 
18. Mumford, D., Shah, J.: Optimal approximations by piecewise smooth functions and associated variational problems. Comm. on Pure and Applied Math. 42 (1989) $577-685$

19. Mory, B., Ardon, R., Thiran, J.P.: Fuzzy region competition: A convex two-phase segmentation framework. In: International Conference on Scale Space Methods and Variational Methods in Computer Vision. (2007) 214-226

20. Brox, T., Cremers, D.: On the statistical interpretation of the piecewise smooth mumford-shah functional. In: Scale Space and Variational Methods in Computer Vision. (2007) 203-213

21. Li, C.M., Kao, C.Y., Gore, J.C., Ding, Z.H.: Implicit active contours driven by local binary fitting energy. In: IEEE Computer Vision and Pattern Recognition or CVPR. (2007) 1-7

22. Kybic, J., Unser, M.: Multidimensional elastic registration of images using splines. In: International Conference on Image Processing. (2000) 455-458

23. Rueckert, D., Sonoda, L.I., Hayes, C., Hill, D.L.G., Leach, M.O., Hawkes, D.J.: Nonrigid registration using free-form deformations: Application to breast MR images. IEEE Trans. Medical Imaging 18 (1999)

24. Bookstein, F.: Principal warps: Thin-plate splines and the decomposition of deformations. IEEE Transactions of Pattern Analysis and Machine Intelligence 11 (1989) 567-585

25. Fornefett, J., Rohr, K., Stiehl, H.: Elastic registration of medical images using radial basis functions with compact support. In: Conference on Computer Vision and Pattern Recognition. (1999) 402-409

26. Babuska, I., Melenk, J.M.: The partition of unity method. International Journal of Numerical Methods in Engineering 40 (1997) 727-758

27. Shewchuk, J.: An introduction to the conjugate gradient method without the agonizing pain. Technical report, Carnegie Mellon University, Pittsburgh, PA, USA (1994)

28. Makram-Ebeid, S., Somphone, O.: Non-rigid image registration using a hierarchical partition of unity finite element method. In: International Conference on Computer Vision. (2007) 